\title{
L'intimité, la fiabilité et les paroles des femmes : quand les violences conjugales rencontrent les institutions
}

L'intimità, l'affidabilità e le parole delle donne: quando le violenze coniugali incontrano le istituzioni

Intimacy, Reliability and Women's Words: When Domestic Violence Meets

Institutions

\section{Alessandra Gribaldo}

\section{OpenEdition}

\section{Journals}

Édition électronique

URL : https://journals.openedition.org/aam/2883

DOI : $10.4000 /$ aam. 2883

ISSN : 2038-3215

Éditeur

Dipartimento Culture e Società - Università di Palermo

Référence électronique

Alessandra Gribaldo, «L'intimité, la fiabilité et les paroles des femmes : quand les violences conjugales rencontrent les institutions », Archivio antropologico mediterraneo [En ligne], Anno XXIII, n. 22 (1) | 2020, mis en ligne le 20 juin 2020, consulté le 21 septembre 2021. URL : http://journals.openedition.org/ aam/2883 ; DOl : https://doi.org/10.4000/aam.2883

Ce document a été généré automatiquement le 21 septembre 2021.

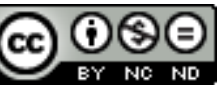

Archivio antropologico mediterraneo è distribuita con Licenza Creative Commons Attribuzione - Non commerciale - Non opere derivate 4.0 Internazionale. 


\title{
L'intimité, la fiabilité et les paroles des femmes : quand les violences conjugales rencontrent les institutions
}

\author{
L'intimità, l'affidabilità e le parole delle donne : quando le violenze coniugali \\ incontrano le istituzioni \\ Intimacy, Reliability and Women's Words: When Domestic Violence Meets \\ Institutions
}

Alessandra Gribaldo

1 La prise de parole des femmes est historiquement centrale pour la possibilité de dénoncer les rapports inégaux de pouvoir entre les genres et de changer, en conséquence, la société. Différentes traditions féministes partagent le même objectif de pouvoir parler de la violence et de la dénoncer. La réintroduction du langage est l'une des principales stratégies utilisées pour aider les victimes de violences et pour faciliter leur rétablissement. L'acte politique consistant à briser le silence incite les femmes à parler, à prendre position, à s'exprimer et, en ce faisant, à nommer la violence. C'est principalement ce discours du pouvoir thérapeutique du récit, que l'on trouve aussi dans des travaux anthropologiques sur la violence (Jean-Klein, Riles 2005 : 178-179), qui identifie les femmes comme des sujets qui doivent s'exprimer et raconter leur histoire. La pratique féministe du dialogue impliquant l'entourage et les soutiens est un outil puissant pour lier le personnel et le politique, en invoquant un espace public partagé qui permet de reconnaître la violence individuelle dans un horizon social de sens.

2 Récemment, même avec ses contradictions, le mouvement international \#metoo a su porter à l'attention sociale les pratiques sexistes de discrimination et abus envers les femmes agies par des hommes qui abusent de leur position de pouvoir (Kipnis 2018). L'action de la dénonciation collective a eu la capacité de soulever la question de la prise de parole individuelle, qui expose chaque femme en la remmenant paradoxalement à 
une condition de jugement et de vulnérabilité. La violence à l'égard des femmes a suscité une attention croissante ces dernières années en Italie. Elle est même devenue une question politique, comme le montre le succès chez les générations les plus jeunes du mouvement féministe Non Una Di Meno [Pas Une De Moins]. Le besoin de dénoncer la violence et le respect de la volonté des femmes sont très présents dans le mouvement et le débat féministes.

3 Le contexte légal, en particulier, a été indiqué comme milieu problématique pour la prise de parole des femmes. Pour pouvoir procéder légalement envers un accusé, il faut une prise de parole exprimée par une seule personne, qui constitue un témoignage contre celle qui a perpétré la violence.

4 Le débat public sur ce thème en Italie remonte aux années 1970 qui s'est surtout focalisé sur le témoignage de la victime de violence sexuelle. Un documentaire diffusé par la télévision publique (la RAI) en 1979, Processo per stupro [procès pour viol], a largement contribué à faire connaître la question du viol et de la justice. Il a ouvert le débat public en donnant à voir une culture qui mettait l'accent sur la moralité de la victime et sur le voyeurisme pornographique du tribunal à l'égard de la déclaration de la témoin. Dans ce procès, les modalités d'exposition d'une jeune femme et la gestion générale de la procédure ont conduit l'avocate de la partie civile, Tina Lagostena Bassi, à formuler une plaidoirie convaincante, aujourd'hui célèbre, qui interpellait le tribunal :

Parce que la défense est sacro-sainte et inviolable, elle est vraie. Mais aucun d'entre nous, les avocats, ne penserait jamais à préparer une défense pour vol comme on le fait pour les violences sexuelles. [...] C'est pourtant la routine : c'est le procès de la femme. [...] Je ne suis pas la défenderesse de la femme, Fiorella. Je suis l'accusatrice d'une certaine manière de mener les procès pour viol' ${ }^{1}$.

5 En Italie, les luttes et les débats féministes ont réussi principalement à avoir un impact sur les pratiques juridiques en matière de violences sexuelles en reformulant considérablement la définition du délit et la façon dont elle est traitée au tribunal (Bossini 2017 ; Pisa 2017).

6 Les violences conjugales ont toutefois une histoire différente de celle des violences sexuelles en ce qui concerne les rapports avec le droit : bien que les luttes contre les violences conjugales soient clairement liées à celles contre les violences sexuelles, dans le cas de la violence entre partenaires, l'accent a été mis sur la création de refuges pour les femmes plutôt que sur le droit lui-même.

7 Je voudrais aborder dans cet article les paroles et témoignages des femmes contre les violences conjugales et leurs relations avec les institutions. Le recours au langage juridique revêt une valeur décisive, effective et symbolique dans la définition de la condition de victime. Le passage au niveau institutionnel apparaît comme une étape attestant que la violence a été perpétrée, que le témoignage de cette violence est véridique et que les femmes sont des sujets politiques. La pression qui s'exerce sur la victime de violences conjugales pour qu'elle parle des faits, d'elle-même et de sa relation avec l'auteur, et pour qu'elle dénonce ce dernier à la justice, a des implications importantes.

8 Le processus d'identification de la violence entre partenaires soulève la question de savoir si la victime est capable d'exprimer l'acte lui-même, de sa propre voix, dans le contexte institutionnel. Comment faire en sorte que la violence qui intervient dans les relations intimes devienne présente dans les institutions? Comment le sujet maltraité 
se constitue-t-il devant elles ? Quelles sont les exigences en ce qui concerne sa capacité à identifier et à dénoncer l'acte violent? En suivant les réflexions de Foucault sur la véridiction, nous pouvons nous demander « comment les sujets sont effectivement liés dans et par les formes de véridiction où ils s'engagent» (2012: 9). Et plus spécifiquement, dans le cas qui nous intéresse : de quelle manière le genre et l'intimité affectent-ils la fiabilité des femmes dans les témoignages de violences conjugales?

\section{Les victimes hésitantes}

9 La littérature sur la violence de genre et le droit a montré que l'expérience de la violence, chez la victime, était exacerbée par la violence systémique de l'ordre social et juridique. Un procès se déroule selon sa propre logique interne, de la déclaration liminaire jusqu'à la décision du juge, en occultant les spécificités du délit dans des contextes intimes et en rejetant l'ambiguïté qui se produit à l'intersection de l'intimité et de la violence ainsi que les implications matérielles des déséquilibres de pouvoir (Boiano 2015).

10 Cette dynamique est manifeste dans les exigences du tribunal en matière de véracité dans le témoignage des femmes qui ont connu des violences conjugales (Ferraro 2006, Merry 2006, Lazarus-Black 2007, Trinch 2003). Dans un contexte où les documents écrits sont rarement disponibles, les témoignages jouent un rôle essentiel. En l'absence d'attestations de consultation aux urgences ou de déclarations de témoins, la preuve repose souvent exclusivement sur le témoignage de la victime. Celui-ci constitue le principal moyen d'établir la preuve, au point que la condamnation peut n'être prononcée que sur cet élément, à condition qu'il soit parvenu à un degré suffisant de validité. Raconter sa propre expérience en termes reconnaissables dans l'espace public ne va pas de soi et n'est pas si facile. Déclarer avoir subi des violences veut dire revendiquer des droits et réclamer un changement en se référant à son histoire personnelle. Témoigner de violences conjugales implique, pour les femmes, le risque de se trouver marginalisées, jugées excessives, perçues comme jouant les victimes et considérées comme des manipulatrices : les femmes ne parlent pas; elles parlent trop ou trop peu; et leurs témoignages sont paradoxaux dans leur constitution même, caractérisés par une structure inadéquate et contradictoire (Gribaldo 2014).

11 Mes réflexions reposent sur un terrain que j'ai mené à Bologne, entre 2010 et 2011, en tant qu'anthropologue impliquée dans une recherche d'équipe multidisciplinaire qui comprenait aussi des sociologues et des criminologues et dont les résultats ont été publiés dans Creazzo (2013). Le projet, sous la direction de la chercheuse en criminologie Giuditta Creazzo, visait à analyser la relation entre les femmes qui ont souffert de violence domestique et le système judiciaire en Italie, Royaume-Uni, Espagne et Roumanie. Des entretiens ont été conduits dans chaque contexte national à la fois avec des femmes victimes de violence, contactées à travers les maisons de refuge, et différentes catégories de professionnels qui travaillent dans la prise en charge sanitaire, légale et sociale de la violence domestique. En outre, on a conduit des observations dans les tribunaux avec le but d'enregistrer les dynamiques lors des procès. Ma recherche, dans la ville de Bologne, a été conduite en collaboration avec la Casa delle donne per non subire violenza, un centre d'écoute et maison de refuge pour femmes. Entre juin 2010 et décembre 2011, j'ai donc réalisé dans ce centre 20 entretiens avec ces femmes et j'ai conduit l'observation de 25 audiences publiques pour des cas 
d'abus familial dans le tribunal d'instance. Les audiences que j'ai pu suivre concernaient d'une manière spécifique le délit de maltrattamenti in famiglia [violence familiale] (art. 572 du Code pénal). Cependant, d'autres délits étaient débattus en même temps: coups et blessures, stalking, insultes, menaces et, dans un cas, tentative de meurtre. Le fait d'observer une audience, planifiée dans une journée, consistait à passer beaucoup de temps au tribunal, à cause de retards et de renvois ; flâner, parler avec les témoins et écouter les procès pour d'autres délits m'ont donné la possibilité de mieux saisir les spécificités des affaires de violence domestique. Tous les entretiens en profondeur, qui ont duré d'une heure et demie jusqu'à trois heures, se sont focalisés en priorité sur les aspects légaux. Toutefois, les entretiens ont même laissé du temps pour des récits autour de la relation avec le partenaire, du paradoxe d'un système souvent jugé par elles à la fois inquisiteur et inefficace. Les voix des femmes abusées que j'ai collectées appartiennent à des personnes qui m'ont été présentées comme "ayant entrepris un chemin ", et donc ayant envie de parler de leurs expériences avec une chercheuse. Par ailleurs, avec Giuditta Creazzo, nous avons réalisé 21 entretiens dans le district de Bologne avec des professionnel-le-s impliqué-e-s dans la gestion des cas de violence domestique : neuf magistrats (procureurs et juges), un avocat, le directeur d'un service d'urgence, six professionnels-le-s du centre antiviolence et du service social de la mairie. Le focus de ces entretiens a porté sur les aspects principaux et récurrents de l'assistance légale et de la sûreté dans la gestion de cas de violence domestique ainsi que sur les dynamiques des audiences et des procès. Les entretiens et les observations dans le tribunal ont été conduits dans la même période ; une superposition qui a permis de souligner le contraste à la fois entre les récits des professionnel-le-s et ce qui se passait dans le tribunal, et entre les déclarations des femmes à l'intérieur et en dehors de celui-ci.

12 Concernant mon travail, ma réflexion a été développée au fils des années (Gribaldo 2014, 2019a, 2019b), aussi grâce à une bourse qui m'a permis de revenir sur mes données ${ }^{2}$. Les pages qui suivent ne peuvent pas tenir compte du complexe débat anthropologique autour des questions légales (Nader 2002, De Lauri 2013), et des régimes de légalité (Resta 2017). Toutefois, elles proposent une analyse ethnographique de la «pratique juridique considérée comme expression d'un savoir juridique incorporé» (Ibidem: 10), et de la relation compliquée des femmes avec la loi et les institutions, «dans des contextes où la violence fait partie de la vie quotidienne» (Mazzucchetti, Piccoli 2016:3).

13 La plupart des femmes avec lesquelles je me suis entretenue au refuge n'avaient pas porté plainte et n'avaient pas engagé de procédure judiciaire. Pour différentes raisons, d'ordre économique, pratique ou sentimental, la victime de violences conjugales peut décider de ne pas intenter de poursuites, ou être prise de doutes et retirer sa plainte: sa relation intime, les enfants qu'elle a eus avec l'auteur et la crainte d'éventuelles conséquences, comme le placement en garde à vue, peuvent également jouer un rôle important.

14 En Italie, il existe une différence cruciale entre le régime d'engagement de poursuite pour le délit de violence sexuelle et celui de violence conjugale. Si la violence sexuelle est poursuivable à la suite d'une dénonciation par la victime, le délit de violence conjugale prévoit, quant à lui, la règle de la poursuite d'office, qui signifie que l'action pénale doit être démarrée au moment où la notice du délit arrive, ce qui implique que 
même si la victime n'a pas déposé la plainte, elle peut être appelée à participer au procès contre son gré.

De plus, la difficulté de s'occuper des délits de violence au sein de la famille est liée au retrait des accusations. Ces rétractations sont d'ailleurs plus complexes que celles qui concernent d'autres délits, précisément parce qu'elles impliquent les relations, la famille et les liens d'affection. Le pourcentage de rétractation en matière de délits liés à la violence conjugale est en effet très élevé en Italie.

Stefania Crocitti, criminologue de l'équipe de recherche, a remarqué en analysant les dossiers du tribunal de Bologne que $61 \%$ des accusations sont retirées au cours des étapes de l'enquête ou du procès (Arcidiacono, Crocitti 2015) 3 $^{3}$ Quand les violences sont classifiées par le parquet comme "maltrattamenti in famiglia", la victime ne peut désister de sa plainte, même si elle peut rétracter, nier ou minimiser les faits dénoncés. Cela n'a pas d'effets sur l'action pénale: la volonté - ou non - de la plaignante de poursuivre l'accusé est, du point de vue formel, sans importance pour la poursuite de celui-ci. Souvent, les victimes produisent une déclaration de désistement d'action, soit devant les carabinieri ou la police, soit par la suite, face au juge qui devra en tenir compte.

Les audiences regorgent d'affirmations du type "Je ne m'en souviens pas"; de silences; de larmes et de gêne; de récits accélérés, brisés par l'émotion, ou d'une tendance à radoter, à s'éterniser sur des aspects qui ne sont pas pertinents d'un point de vue juridique ; de contradictions; et d'omissions. Les efforts déployés par le juge et le procureur pour organiser le compte rendu des femmes dans un récit plausible et cohérent finissent par créer des souffrances, des difficultés, davantage de confusion et un sentiment d'impuissance chez la témoin. J'ai constaté pendant les audiences une tendance générale à normaliser la violence par la banalisation du conflit familial, la défense tendant à utiliser un langage simplifié et aveugle au genre. La défense remplaçait systématiquement, par exemple, des mots comme mots "violence", voire "conflit", par "chamaillerie", "bagarre " ou "situation difficile", et utilisait fréquemment des expressions impersonnelles pour décrire les épisodes de violence ( il y a eu des gifles »), banalisant l'événement violent et niant sa dimension de genre. Alors que le crime d'honneur a été aboli en Italie en 1981, la jalousie reste un thème pertinent dans les procès pour violence. La défense cherche en particulier à souligner l'aspect " humain " et banal de la jalousie, en l'évoquant explicitement comme " une question éthique, et non juridique». Les jugements font apparaître une tendance générale à sous-estimer la violence, à nier sa persistance et à discréditer les paroles des femmes en l'absence de preuve. Le tableau est encore compliqué par la longueur des procédures du système judiciaire italien et par la pratique bien établie qui consiste à accorder des circonstances atténuantes, et par conséquent une condamnation avec sursis, aux accusés sans casier judiciaire. Les magistrats honoraires (procureurs adjoints) représentent le ministère public dans les audiences pour violences conjugales. Ils changent souvent d'une audience à l'autre, ignorent le détail des pièces du dossier de l'enquête préliminaire et ne sont pas experts en la matière.

Il faut rappeler que la femme victime de violence conjugale est suivie par un avocat seulement si elle se constitue partie civile. Les femmes qui décident de ne pas le faire se retrouvent au tribunal sans y être bien préparées. Le procureur ne vérifie pas systématiquement s'il existe d'autres chefs d'accusation. Si l'avocat de la victime ne les a pas toutes rassemblées dans le même dossier, les accusations multiples qui ont été 
portées au fil du temps contre le partenaire finissent facilement dans les mains de procureurs différents et sont traitées dans des procédures séparées.

L'ambivalence inhérente à l'intimité de la relation entre la femme et l'auteur des violences détermine souvent la dynamique de la procédure juridique : lorsque, au cours d'une audience, la défense a demandé à la plaignante si la menace proférée par l'accusé de la tuer avec des ciseaux et de les retourner ensuite contre lui, pour tomber à genoux devant elle, constituait un "geste théâtral pour démontrer ses sentiments d'amour ", cette dernière, très tendue, lui a répondu par l'affirmative. Elle n'a souri à son jeune avocat que lorsque le juge a prononcé une condamnation avec sursis, ainsi que l'avait expressément demandé l'accusation (notes de terrain, janvier 2011). Le procès peut être vécu comme une épreuve pour la partie lésée parce qu'il constitue la révélation publique d'une relation intime et de son échec. L'exposition de la femme en tant que victime de violence, la rencontre potentielle avec son ex-conjoint et les jugements possibles sur ses capacités en tant que conjointe et en tant que mère contribuent en outre à sa détresse potentielle.

Dans l'étude ethnographique qu'elle a menée à la Trinité, Lazarus-Black a identifié les rites de la cour qui sont présents dans la salle d'audience et qui peuvent être considérés comme des « événements stylisés de façon profane » $(2007: 92)$ en matière de violences conjugales. Ils expriment un pouvoir qui se reproduit et qui comporte des dispositifs d'intimidation, d'humiliation, d'objectivation, de silence et de pouvoir discrétionnaire. Dans une affaire que j'ai observée, la défense a demandé à la plaignante de confirmer qu'elle avait eu plusieurs enfants de trois partenaires différents et qu'elle s'était enfuie de chez elle alors qu'elle était enceinte, quand elle avait dix-sept ans, puis l'a interrogée sur ses contacts avec les services sociaux et sur ses relations avec ses parents et ses frères et sœurs (la défense : «Je ne veux pas vous faire passer un interrogatoire serré, mais vous êtes la seule témoin contre l'accusé ». Notes de terrain, février 2011).

Le recours à l'accusation de maltrattamenti in famiglia pose pourtant d'autres problèmes que le manque de reconnaissance, la sous-évaluation de la violence, l'hésitation de la victime à demander justice ou les peines trop légères. La façon dont se produit le manque de reconnaissance est plus complexe qu'un simple mépris ou préjugé, ou un manque d'attention et de sensibilité au contexte à l'origine de la violence. Les modalités de la procédure judiciaire soulèvent en réalité un certain nombre de questions quant à la possibilité d'obtenir la vérité du sujet parlant. Le thème du dépôt de plainte contre le partenaire ou l'ex-partenaire est l'un des aspects les plus complexes des récits des victimes ; il est lié à une série de choix, de jugements et de comportements.

Tandis qu'elle décrivait ses difficultés avec le processus judiciaire, une jeune femme a ainsi déclaré que le droit devait l'emporter sur les choix de la victime, et prendre le pas sur son propre choix : si le droit fonctionne, c'est seulement parce qu'il est dissocié de la décision de la victime («La police devrait enclencher tout le processus, même s'il ne vous a frappée qu'une fois. Cela doit être jugé, même si la femme dit non $\left.{ }^{4} »\right)$. Mais si le droit sait, agit, parle pour la victime et procède d'office, il est voué à l'échec : cette même femme a admis que, dans son propre cas, le procès avait échoué, alors même qu'un responsable de la police avait insisté pour qu'elle fasse appel à la justice ( « Il a réussi à faire porter l'affaire devant le tribunal, bien que j'aie dit : "Non, je ne veux pas l'accuser, non, je ne veux pas de procès". Il a réussi à faire en sorte que l'affaire passe au tribunal. Mais ensuite, au procès, le juge l'a acquitté parce que je n'ai pas témoigné »). 
23 En théorie, la forme rituelle du procès légal - par sa structure et ses modalités - devrait protéger l'accusé (et la victime qui parle) plutôt que la pratique commune des services sociaux. Garapon nous suggère une interprétation du sens des formalités de la loi :

Le rituel judiciaire, ses formes, son jeu, son extériorité ont quelque chose de plus respectueux pour l'accusé que le monde apostolique du travail social. Ne vaut-il pas mieux demander à un accusé de jouer la comédie que d'envahir son 'jardin secret'? N'est-ce pas faire violence à quelqu'un que de prétendre le comprendre malgré lui? (1997: 263).

Cependant, la logique du système qui cherche à prouver le délit est intrinsèquement violente envers les femmes qui ont souffert de la violence domestique: plus l'évaluation rentre dans leurs témoignages, moins elle devient convaincante. Le procureur et le juge soulèvent des questions et des remarques qui visent à encadrer le témoignage des témoins, notamment de la partie lésée. À partir de l'incapacité de la victime de porter plainte, on cherche à tracer le délit en certifiant l'expérience de la victime, afin qu'elle devienne une prise de parole au sens plus large. Or, l'expérience continue d'être en quelque sorte non intelligible.

Le témoignage est central, même dans les cas où l'accusation a été abandonnée. Le passage qui suit, tiré d'une audience, illustre précisément ce point : la témoin, à côté de laquelle j'étais assise, n'a pas cessé d'exprimer son mécontentement par des gestes et commentaires pendant le témoignage de son enfant, qui disculpait le père et l'exconjoint. Lorsqu'elle a été appelée à témoigner par le juge, elle a pourtant fait part de son hésitation, exprimé son pardon et évoqué ses sentiments. Elle ne voulait pas continuer, alors elle a retiré sa déclaration :

Avocat de la défense : Pourquoi, le jour X, avez-vous retiré votre plainte?

Témoin : Tout était fini.

A : Qu'est-ce que vous voulez dire?

$\mathrm{T}$ : Tout était fini. Parce que le tribunal ne servait plus à rien... [...]

A : Votre mari vous a-t-il battue ?

$\mathrm{T}$ : Non, non. Des claques, quelquefois, des coups, de grosses claques, mais battue jamais de façon violente, non, jamais... [...]

Juge : Combien de fois votre mari a-t-il battu les enfants?

$\mathrm{T}$ : Pas très souvent. Ce n'est pas un ivrogne ou qui a de mauvaises habitudes ou des attitudes bizarres. C'est quelqu'un de fort, déterminé. On s'est disputés aussi, à cause de la jalousie... On s'aimait... [Sourires]

$\mathrm{J}:$ Une fois par semaine?

$\mathrm{T}$ : [Évasive] Moi aussi, quelquefois. J'ai giflé les enfants plusieurs fois.

(Notes de terrain, octobre 2010, Bologne)

La victime, les travailleurs sociaux, l'équipe juridique, la police, mais aussi les avocats du refuge pour les femmes, reconnaissent en général qu'une "revictimisation" se produit pendant les procès. Dans ces affaires, tout ce que Garapon (1997) attribue à l'accusé dans son archéologie du cadre judiciaire du droit civil - les états d'aliénation, d'infériorité et de désorientation, les attentes relatives au respect des règles de la salle d'audience, l'impossibilité fondamentale d'utiliser des mots qui ont suffisamment de poids - se retrouve chez la victime.

La question du respect des silences est un élément problématique, souvent abordé dans les débats qui se tiennent au refuge pour les femmes de Bologne, où l'on insiste souvent sur l'impossibilité de dire et sur le choix de ne pas parler, en tout cas dans un cadre public et juridique. De nombreuses discussions portent aussi sur la façon dont les travailleurs du refuge parviennent à gérer leur frustration quand ils sont confrontés à 
une personne qui retire sa plainte, qui ne revient pas au refuge ou qui ne répond pas au téléphone, bref, qui disparaît. Les femmes sont encouragées à parler dans des espaces protégés; elles sont invitées à partager leur expérience avec les travailleurs du refuge et avec d'autres femmes qui se trouvent dans des situations similaires. Au cas où elles décideraient de porter plainte, les avocats du refuge sont disponibles pour les aider. Inversement, les responsables des services sociaux considèrent que la voie judiciaire va de soi, qu'elle constitue une exigence essentielle - bien qu'elle ne soit pas techniquement nécessaire - pour celles qui veulent utiliser les services, dans la mesure où elle atteste la violence devant les institutions et indique, en outre, que les femmes prennent leurs responsabilités. Cette insistance découle précisément de l'ambivalence des femmes quant à leurs compagnons, comme le soutient un travailleur social : « elles déposent une plainte, puis la retirent; elles virent leurs conjoints, puis les laissent revenir ; c'est très compliqué ${ }^{5}$ ». Les travailleurs sociaux ne demandent pas à être persuadés, mais ils demandent aux femmes de garantir qu'elles ne disparaîtront pas, qu'elles s'en tiendront à une conduite et qu'elles sont globalement dignes d'être prises en charge par les services. Les exigences des institutions envers les femmes victimes de violence reproduisent en conséquence l'ambivalence et produisent simultanément deux messages contradictoires : d'un côté, les services sociaux demandent à la femme de porter plainte dans tous les cas, afin d'accéder aux services, tandis que, de l'autre, le système judiciaire attend de leurs accusations qu'elles constituent plus et autre chose qu'un moyen au service d'une fin, que les femmes s'engagent pleinement dans la procédure. La contradiction relève du fait qu'on demande aux femmes de porter plainte dans un but instrumental - de fait, pour pouvoir être suivie plus aisément par les services sociaux - et qu'en même temps, la loi exige que la femme reste « contrainte » à cette plainte par la poursuite d'office car la procédure continue indépendamment de l'éventuelle rétractation de la victime.

Les professionnels du droit et du travail social ont insisté constamment, au cours de mes entretiens, sur le fait que la plaignante devait être encouragée pour être au clair avec elle-même. Ce manque de clarté et de détermination - perceptible dans les positions et déclarations des femmes qui ont subi des violences de la part de leurs partenaires - est connu dans la littérature: les professionnels expriment cette frustration en posant la question freudienne infâme et finalement sans réponse : "Que veulent les femmes?". Les victimes de violences conjugales apparaissent soit trop difficiles soit trop peu coopératives : «il n'y a aucun moyen de savoir » ce que veulent réellement les victimes (Mills 2006: 48).

Dans le contexte italien, c'est l'acte de porter des accusations qui fait de la femme une victime consciente, suffisamment fiable pour intenter une action en justice. Denunciare [porter plainte] veut dire prendre la parole en tant que sujet de droit afin de réclamer justice, mais cela implique aussi de s'exprimer, de s'exposer en public et de rendre l'affaire explicite, autrement dit de prendre position. Comme le remarque Bourdieu (1988 : 17), en suivant Benveniste, les mots qui servent à dire le droit dans les langues indo-européennes ont leurs racines dans le verbe «dire », parce que le «dire droit» implique des énoncés performatifs sur le pouvoir de l'institution qui confère une autorité à ces énoncés. Le thème du récit de la violence, de la prise de parole et du dépôt de plainte apparait constamment dans les cas de violences conjugales, parce que les femmes sont incitées à reconnaître et à nommer le coupable. Ce message a été véhiculé récemment par les campagnes contre la violence de genre : les campagnes sur la violence contre les femmes n'en sont qu'à leurs débuts en Italie, contrairement aux 
pays anglo-américains; elles ont été lancées en réponse aux conclusions de la première étude statistique nationale. La campagne nationale de 2013, intitulée Riconosci la violenza (Reconnais la violence), montrait par exemple la photographie d'un visage d'homme couvert par le slogan: "La violence prend plusieurs formes. Apprends à la reconnaître. Un homme violent ne mérite pas ton amour. Il mérite une plainte ${ }^{6}$ ». Parler de violence revient, en ce sens, à porter plainte ; c'est-à-dire l'acte de nommer la violence, effectué par un sujet constitué par des conceptions normatives de la puissance d'agir. Indiquer l'auteur, prendre la parole et agir (quitter le partenaire violent) sont autant d'éléments qui convergent dans un but pédagogique.

Les actions des forces de l'ordre visent à soutenir la personne dans sa détermination à poursuivre l'action en justice - en reconnaissant son hésitation, en la comprenant, en vérifiant qu'elle est suffisamment forte et qu'elle est capable de "maintenir " ses accusations - et à obtenir d'elle toute l'histoire. C'est ce qui ressort des mots d'un brigadier des carabiniers, lorsqu'il expliquait les difficultés d'intervention dans les cas de violences conjugales et le besoin de créer un climat de confiance mutuelle («Elles ne veulent pas raconter [l'histoire]. Mais comment pouvons-nous préparer une intervention s'il n'y a pas d'histoire ${ }^{7}$ ?»). Pour “performer" le sens du dépôt de plainte, à savoir une demande de jugement, les femmes doivent dire le tout des violences qu'elles ont subies et de leurs relations avec leurs auteurs. Au cours de mes entretiens, les travailleurs sociaux s'accordaient à considérer qu'il était crucial que les femmes s'expriment en portant plainte. À partir de l'accusation, comme l'expliquait un responsable des services sociaux, la voie est particulièrement cahoteuse et humiliante ; elle implique une capacité à "surmonter les épreuves". Malgré cette conscience de la nature problématique du dépôt de plainte, les professionnels admettent avec réticence que cette étape fait une grande différence dans la manière dont les services sociaux prennent en charge une affaire spécifique, parce qu'elle affecte le degré de crédibilité qu'ils attribuent à la victime des violences :

Après le dépôt de plainte, la voie est particulièrement complexe: les interrogatoires, pour vérifier si c'est vrai ou faux, si nous avons fait tout le nécessaire [si] elle est allée aux urgences, et si elle a obtenu un certificat ou non. Donc, souvent, les choses que nous donnons en retour à ces femmes les punissent au lieu de les aider. [...] Nous les croyons plus si elles ont porté plainte [soupir] parce qu'elles ont fait une chose difficile, à savoir qu'elles ont agi, et que ce n'est pas instinctif. C'est d'une certaine manière moins instinctif que de se contenter de dire : "Il m'a frappée" ou "Il me frappe".

Les travailleurs sociaux doivent porter un jugement sur la femme, contribuer à confirmer la véracité de ses déclarations et prendre position sur les faits et la vérité de ce qu'elle dit. Au cours de mes entretiens, une femme a qualifié les services sociaux de «bras du juge en dehors du tribunal ». Parler aux institutions de la violence qu'elle subissait de la part de son conjoint, c'était faire entrer sa biographie personnelle dans un code moral préexistant. Ce code et la subjectivité de la victime - qui est requise sont étroitement liés au délit de "violence familiale". La contradiction entre la nécessité de donner à une femme la possibilité de ne pas porter plainte, le besoin de le faire pour qu'elle soit prise en charge par les services sociaux, la responsabilité ultime de ses propres décisions et l'image de la victime ayant besoin de protection contre un contexte hostile et contre ses propres choix se reflète dans la manière problématique dont le Code pénal décrit les délits commis contre les femmes. 


\section{Le sujet victime de violences conjugales et le droit}

L'article 572 qui prévoit la poursuite d'office correspond à un besoin évident, ressenti à l'époque de la rédaction du «Code Rocco », le Code pénal écrit en époque fasciste, qui, bien que reformé au fil du temps dans ses parties plus anachroniques, est encore en vigueur : protéger la victime qui portait plainte dans un contexte où elle était supposée soumise à une pression exceptionnelle pour retirer ses accusations de la part de la famille et du milieu social. De toute évidence, les "meilleures intentions" du droit étaient déconnectées d'une politique de changement social en matière de liberté des femmes, et cela a produit un sujet des droits par définition non autonome: un minus habens qui a littéralement besoin de l'État pour tenir sa position.

L'article du Code pénal qui s'appelait auparavant maltrattamenti in famiglia o verso fanciulli [mauvais traitements en famille ou envers des enfants] a été modifié en 2013 pour répondre à la sensibilisation croissante à la question de la violence genrée 9 . Pourtant, la nature et la structure du délit, ainsi que la caractéristique essentielle du régime de légalité des poursuites - qui ne s'applique pas en cas de délits sexuels - ne sont pas modifiées pour l'essentiel.

En général, la logique de la loi sur les violences conjugales témoigne du souci d'éliminer l'ambivalence. Cela implique que, dans les cas de violences entre partenaires, la rétractation est beaucoup plus fréquente que dans les autres délits: la réticence de femmes à porter des accusations, et leurs possibles réserves quant au fait d'aller jusqu'au bout du processus judiciaire - qui se traduisent par des abandons et des rétractations - n'est pas traitée par des actions de soutien aux femmes, mais par un processus de décision institutionnel qui ne tient pas compte de ces réserves (Virgilio 2016). La protection judiciaire des femmes victimes de violence suppose que les femmes sont soit des sujets libres, soit des agents faibles et passifs : une telle dichotomie n'existe nulle part ailleurs dans le Code pénal. La logique qui sous-tend la catégorie des délits poursuivis d'office conçoit le sujet qui a subi les violences de manière spécifique. Ce ne sont pas seulement les critères de gravité du délit qui exigent des poursuites, mais aussi la définition de la victime - dont la subjectivité est remplacée par l'État dans les délits qui font l'objet de poursuites d'office -, parce que ses décisions ne peuvent être considérées comme fiables. La règle des poursuites d'office indique clairement que le droit sait mieux que les «femmes battues".

Cette exigence de parler de soi-même - dire la vérité de soi-même, soit à la suite de violences soit en raison d'éventuelles futures violences - a été largement explorée dans l'anthropologie des réfugiés (Beneduce 2015; Malkki 1996; McKinley 1997; Sorgoni 2019, parmi d'autres). Fassin a souligné le paradoxe qui sous-tend l'exigence que les réfugiés apportent la preuve de leur persécution dans leur pays d'origine: l'augmentation du taux de refus des demandes d'asile sert de défense à ce chiffre. Plus les magistrats se méfient des preuves de persécution potentielle ou réelle présentées par les requérants, plus le principe de l'asile prend de la valeur (Fassin 2013).

Ce déplacement de l'attention - de la véracité du récit à la sincérité de la personne n'intervient pas seulement dans les audiences des réfugiés, mais aussi, en Italie, dans les procès pour violences conjugales. Dans le cas des réfugiés, l'État reconnaît la violence passée, liée à un autre contexte politique territorial, alors que dans le cas des violences conjugales, il demande la preuve de la violence subie de la part du partenaire dans la vie quotidienne du territoire familial. Dans les deux cas, les victimes doivent 
témoigner de manière irréfutable et vérifiable qu'elles ont fait l'objet de violences pour devenir un sujet ayant le droit d'être reconnu et protégé et de recevoir une indemnisation appropriée. La difficulté de créer un lien entre la violence contre les femmes et les droits humains est due à la nécessité de défendre le statut des droits humains comme un canon dans lequel l'État est appelé à intervenir (Charlesworth 1999). Si les tribunaux ressentent le besoin de soumettre la victime à un tel examen, c'est à cause de l'idéal de la victime en tant que principe abstrait, évoquant l'imaginaire de l'authentique victime de violences méritant d'être défendue.

L'acte de prendre la parole - signaler l'incident et exprimer son expérience en tant que victime de violences - est largement salué comme un élément politique et progressiste. Pour autant, la manière dont les histoires de violence peuvent et doivent être rendues publiques ne se limite pas à la rupture du silence (Fusaschi 2013, Ribeiro Corossacz 2018). L'idée que parler clairement et porter plainte sont les seuls moyens d'expression crée sa propre violence. Lorsque la violence s'exprime, comme le souligne Veena Das, les frontières entre "dire » et "montrer » doivent être protégées de l'imaginaire du dévoilement et de la découverte :

On considère souvent comme la tâche de l'historiographie de briser le silence que trahissent les zones de tabou. Il y a même quelque chose d'héroïque dans l'image de donner aux femmes la parole et de parler pour ceux qui n'ont pas de voix. [...] [Q]uand on utilise une image comme briser le silence, on peut finir par utiliser notre capacité à "déterrer" des faits cachés comme des armes (Das 2007 : 57).

Dans ce cadre, témoigner de la souffrance et être perçu comme étant à l'écoute ont été identifiés comme les objets privilégiés d'un langage socio-politique contemporain dominé par un «ethos compassionnel». Le recours à ce langage a augmenté soudainement ces dernières années (Fassin, Rechtman 2007). Les sujets apprennent à exprimer leur être intérieur en mots et en images à travers différents mécanismes de gouvernementalité qui entremêlent les politiques publiques et les discours psychopédagogiques (Rose 1989). Ces mécanismes impliquent aussi une exposition aux modes d'expression populaires et de divertissement qui tournent autour de ce qui est essentiellement une accumulation de discours sur le soi ; le malaise et la souffrance ; et l'exploration de l'intimité des relations. Le contexte juridique qui entoure le témoignage des victimes de violence entre partenaires dans les tribunaux est manifestement affecté par ces modes d'expression contemporains particuliers qui mettent en jeu les différences de genre, la présentation et l'affichage de soi, la preuve de l'authenticité et les appels à la compassion. Le rapport à la parole se révèle crucial : les fantasmes, règles et obligations tacites qui sous-tendent l'intimité se révèlent quand l'intimité devient un enjeu, et exigent une éloquence analytique/thérapeutique (Berlant 1998 : 287).

39 Si la femme veut être reconnue comme victime, elle doit se constituer elle-même comme une victime et conférer de la réalité au délit par un discours qui fait d'elle un sujet cohérent. La personne qui est à l'origine de l'action en justice parle donc pour l'auteur des violences, parce qu'elle a eu une relation intime significative avec lui et parce qu'il se trouve lui-même déchargé, juridiquement, de l'obligation de parler. Il ne peut parler, quant à lui, que par l'intermédiaire de la défense, ce qui produit son intériorité en tant qu'accusé - une intériorité qui a moins de poids, parce qu'elle est retirée du droit (la jalousie est une question éthique et non juridique). Le fait qu'il revienne à la victime d'évoquer l'acte violent libère l'auteur de sa responsabilité de culpabilité, c'est-à-dire que cela le libère de la position de devoir répondre à 
l'interpellation du droit. L'intériorité décisive et légitime est celle de la plaignante en tant que sujet de droit (la victime de violences a le droit de parler). Il est nécessaire que, dans son aveu, elle joue son rôle de femme soumise, adhérant à ce qu'elle est. Un passage des réflexions de Foucault sur l'aveu est éclairant à cet égard :

l'aveu n'est pas simplement une constatation à propos de soi-même. C'est une sorte d'engagement, mais un engagement bien particulier : il n'oblige pas à faire telle ou telle chose ; il implique que celui qui parle s'engage à être ce qu'il affirme qu'il est, et précisément parce qu'il est cela (Foucault $2012: 5$ ).

Le gouvernement de soi et la réflexivité rendent le témoignage intelligible et lui donnent une valeur juridique; ils font des femmes des victimes fiables. Paradoxalement, cependant, les violences exercées dans une relation intime fournissent rarement une victime aussi parfaite: le sujet fiable qu'exigent les institutions ne correspond pas aux expériences, aux positions, aux décisions et aux hésitations des femmes.

\section{Conclusions}

41 Les institutions qui exigent que la victime assume personnellement la double responsabilité de connaître sa situation et de l'exprimer au cours du procès sont toutes impliquées dans les violences conjugales: le système judiciaire, la médecine et la psychiatrie. La femme - en tant qu'elle est la victime - est la personne qui possède la clé d'accès à elle-même : la conscience de la violence qu'elle a subie est la condition de sa liberté et, par conséquent, de sa capacité à devenir un sujet doté d'une puissance d'action, à découvrir la vérité et donc à la démontrer.

La forme institutionnelle apparaît, en Italie et ailleurs, comme un espace peu capable de prendre en compte la violence de genre dans toute sa complexité. Dans leur neutralité revendiquée, et donc leur autoréférentialité, les services sociaux et le système judiciaire se révèlent être des institutions qui ne reconnaissent pas la nature de la violence entre partenaires.

Les entretiens avec les travailleurs sociaux et les policiers ont donné une image contradictoire des femmes battues (elles sont victimes ambivalentes, elles ne savent pas ce qu'elles veulent, elles sont évasives, intimidées, stratégiques). Celles avec les professionnels légaux ont souligné le manque de preuves, la centralité de leurs déclarations et la nécessité d'être convaincus. Le travail ethnographique a saisi les indices ambigus des logiques de la loi (la déclaration de la violence soufferte et le conséquent jugement de l'accusé) : les silences des femmes dans le tribunal et face aux institutions, le regard perplexe entre le juge et le procureur public devant ces silences, les sourires gênés des juges, les soupirs de travailleurs sociaux.

La victime de violences conjugales n'est pas un sujet que les institutions attendent, et les paroles, les histoires et les témoignages des femmes apparaissent souvent inadaptés dans leur essence même. L'histoire personnelle et la relation de genre deviennent le lieu d'identification du délit sur le plan juridique. La tentative de contextualisation des événements violents, l'analyse approfondie de l'histoire de la relation et de la vie réelle conduit à l'étude potentiellement infinie des expériences et de la subjectivité de la victime. La preuve devient l'expérience de vie de la victime, prise dans des contingences qu'il est impossible de formaliser; mais, en outre, l'ambiguïté des 
sentiments, la sous et la surdétermination de la victime finissent par être un excès que le droit doit prendre en compte, mais qu'il ne peut traiter.

L'ethnographie et la pensée anthropologique peuvent offrir une chance de mieux comprendre et de reconnaître non seulement les femmes qui déclarent leur vérité, mais aussi les femmes - les plus nombreuses - qui, au contraire, ne veulent pas parler devant la justice, qui se rendent invisibles, parce qu'elles ne reconnaissent pas les institutions et ne sont pas reconnues par elles, et qui continuent de hanter la justice.

\section{BIBLIOGRAPHIE}

Arcidiacono D., Crocitti S., 2015 « Criminal Justice System Responses to Intimate Partner Violence : The Italian Case », in Criminology \& Criminal Justice, XV (5) : 613-632.

Beneduce R., 2015 « The Moral Economy of Lying : Subjectcraft, Narrative Capital, and Uncertainty in the Politics of Asylum », in Medical Anthropology, XXXIV (6) : 551-571.

Belmonti M. G., 1980 Un processo per stupro : Dal programma della Rete Due della Televisione Italiana, Einaudi, Torino.

Berlant L., 1998 « Intimacy : A Special Issue », in Critical Inquiry, XXIV (2) : 281-288.

Boiano I., 2015 Femminismo e processo penale, Ediesse, Roma.

Bosini L.E., 2017 « Le proposte di legge in materia di violenza sulle donne all'inizio del dibattito italiano (1979-1980) », in S. Feci, L. Schettini (a cura di), La Violenza contro le donne nella storia. Contesti, linguaggi, politiche del diritto (Secoli XV-XXI), Viella, Roma : 197-212.

Bourdieu P., 1982 Ce que parler veut dire. L'économie des échanges linguistiques, Fayard, Paris.

Charlesworth H., 1999 « Feminist Methods in International Law », in The American Journal of International Law, XCIII (2): 379-394.

Creazzo G. (a cura di), 2013 Se le donne chiedono giustizia. Le risposte del sistema penale alle donne che subiscono violenza nelle relazioni di intimità: ricerca e prospettive internazionali, Il Mulino, Bologna.

De Lauri A. (a cura di), 2013 Antropologia Giuridica. Temi e prospettive di ricerca, Mondadori, Milano.

Das V., 2007 Life and Words : Violence and the Descent into the Ordinary, University of California Press, Berkley.

Fassin D., 2013 « The Precarious Truth of Asylum », in Public Culture, XXV (1) : 39-63.

Fassin D., Rechtman R., 2007 L'Empire du traumatisme. Enquête sur la condition de la victime, Flammarion, Paris.

Ferraro K., 2006 Neither Angels nor Demons: Women, Crime, and Victimization, Northeastern University Press, Lebanon (NH).

Foucault M., 2012 Mal faire, dire vrai. Fonction de l'aveu en justice. Cours de Louvain, 1981, Presses Universitaires de Louvain, Louvain. 
Fusaschi M., 2013 « Le silence se fait parole : ethnographie, genre et superstes dans le postgénocide rwandais », in Archivio Antropologico Mediterraneo, XVI (2) : 29-40.

Garapon A., 1997 Bien Juger. Essai sur le rituel judiciaire, Odile Jacob, Paris.

Gribaldo A., 2014 « The Paradoxical Victim. Intimate Violence Narratives on Trial in Italy », in American Ethnologist, XLI (4) : 743-756.

Gribaldo A., 2019a « Hashtags, testimonies, and measurements. Gender violence and its interpretation », in Anuac, VIII (1) : 7-30.

Gribaldo A., 2019b « The burden of Intimate Violence. Evidence, Experience, and Persuasion », in PoLAR: Political and Legal Anthropology Review, XLII (2) : 283-297.

Istituto Nazionale di Statistica (Istat), 2015 La violenza contro le donne dentro e fuori la famiglia, Istat, Roma.

Jean-Klein I., Riles A., 2005 « Introducing Discipline : Anthropology and Human Rights Administrations », in PoLAR : Political and Legal Anthropology Review, XXVIII (2) : 173-202.

Kipnis L., 2018 « Has \#MeToo Gone Too Far, or Not Far Enough? The Answer is Both », in Where Freedom Starts. Sex Power Violence \#MeToo, Verso Books, London, New York.

Lazarus-Black M., 2007 Everyday Harm : Domestic Violence, Court Rites, and Cultures of Reconciliation, University of Illinois Press, Urbana.

Malkki L. H., 1996 « Speechless Emissaries : Refugees, Humanitarianism, and Dehistoricization », in Cultural Anthropology, XI (3) : 377-404.

Mazzocchetti J., Piccoli E., 2016 « Défis méthodologiques, éthiques et émotionnels d'une ethnographie de l'intime, des silences et des situations de violences ", in Parcours anthropologiques [En ligne], XI, consulté le 03 mai 2019. URL : http:// journals.openedition.org/pa/471; DOI : 10.4000/pa.471.

McKinley M., 1997 « Life Stories, Disclosure and the Law », in PoLAR : Political and Legal Anthropology Review, XX (2) : 70-82.

Merry S.E., 2006 Human Rights and Gender Violence: Translating International Law into Local Justice, University of Chicago Press, Chicago.

Mills L. G., 2006 Insult to Injury : Rethinking Our Responses to Intimate Abuse, Princeton University Press, Princeton.

Nader L., 2002 The life of the law: anthropological projects, University of California Press, Berkley.

Pisa B., 2017 « Il Movimento di liberazione della donna e il primo Centro contro la violenza sulle donne ", in S. Feci S, L. Schettini (a cura di) La Violenza contro le donne nella storia. Contesti, linguaggi, politiche del diritto (Secoli XV-XXI), Viella, Roma : 173-195.

Resta P., 2017 « Diritti negoziati. Trasformazioni sociali e pratiche giuridiche », in EtnoAntropologia, V : 9-22.

Rose N., 1989 Governing the Soul. The Shaping of Private Self, Free Association Books, London.

Ribeiro Corossacz V., 2018 « The Uses of Silence. Researching Sexual Harassment against Female Domestic Workers in Brazil », in Anuac, VII (1) : 43-65.

Sorgoni B., 2019 «What Do We Talk about When We Talk about Credibility? Refugee Appeals in Italy », in N. Gill, A. Good (eds) Asylum Determination in Europe: Ethnographic Perspectives, Palgrave Macmillan, Basingstoke, UK : 221-240. 
Trinch S., 2003 Latinas' narratives of domestic abuse: Discrepant versions of violence, John Benjamins Publishing Corporation, Amsterdam.

Virgilio M., 2016 « Nuovo lessico per il diritto penale : le vittime della violenza di genere contro le donne ", in M. Mantovani, et al. (a cura di), Scritti in onore di Luigi Stortoni, Bononia University Press, Bologna.

\section{NOTES}

1. Extrait de l'argument de Tina Lagostena Bassi dans le procès pour le viol d'une femme de dixhuit ans, Latina, 1978 (Belmonti 1980).

2. En 2016-2017, j'ai reçu l'Independent Scholar Fellowship par l'Independent Social Research Foundation (ISRF).

3. En Italie, $12,2 \%$ seulement des femmes dénoncent les violences conjugales, et $29,6 \%$ seulement les perçoivent comme un délit. Les statistiques nationales indiquent que 13,6\% des femmes ont fait l'objet de violences physiques ou sexuelles de la part d'un conjoint actuel ou d'un ex-conjoint et que $26,4 \%$ ont subi des violences psychologiques de la part de leur conjoint actuel (Istat 2015).

4. Entretien avec Paola, Casa delle donne, Bologne, juillet 2010.

5. Entretien avec un travailleur social, Bologne, juin 2011.

6. http://www.riconoscilaviolenza.it/ consultée le 20 décembre 2019.

7. Entretien avec le brigadier du poste de carabiniers. Ville métropolitaine de Bologne, mars 2011.

8. Entretien avec un responsable des services sociaux, Bologne, juillet 2011.

9. Le décret de loi de 2013 rend obligatoire d'informer la victime de violence en cas de requête d'abandon ou de renouvèlement de l'ordonnance restrictive envers l'agresseur, ou de sa détention préventive, avant le jugement définitif, Il établit aussi que les investigations préliminaires ne peuvent pas durer plus d'un an et que les victimes d'abus ont le droit à l'assistance judiciaire par l'état, indépendamment de leurs revenues, comme pour les peines plus sévères et les mandats de dépôt dans le cas de flagrant délit.

\section{RÉSUMÉS}

Je voudrais aborder dans cet article les paradoxes qui ressortent quand les mots des femmes maltraitées rencontrent les institutions et la loi. Dans un contexte de violence conjugale où les documents écrits sont rarement disponibles, les témoignages jouent un rôle essentiel. Porter plainte et s'exprimer contre la violence signifie prendre la parole en tant que sujet de droit afin de réclamer justice. Le recours au langage juridique revêt donc une valeur décisive, effective et symbolique dans la définition de la condition de victime. Néanmoins, la pression qui s'exerce sur la victime de violences conjugales pour qu'elle parle des faits, d'elle-même et de sa relation avec l'auteur, et pour qu'elle dénonce ce dernier à la justice, a des implications importantes. Ici, à partir d'une recherche menée en Italie je réfléchis à comment faire en sorte que la violence qui intervient dans les relations intimes devienne présente dans les institutions et comment le sujet maltraité se constitue-t-il devant elles. Le gouvernement de soi et la réflexivité rendent le témoignage intelligible et lui donnent une valeur juridique; ils font des femmes des victimes 
fiables. Paradoxalement, cependant, les violences exercées dans une relation intime fournissent rarement une victime aussi parfaite : le sujet fiable qu'exigent les institutions ne correspond pas aux expériences, aux positions, aux décisions et aux hésitations des femmes.

In questo articolo desidero analizzare i paradossi che emergono quando le parole delle donne maltrattate incontrano le istituzioni e la legge. In contesti di violenza nelle relazioni di intimità spesso non sono disponibili prove e la testimonianza delle donne gioca un ruolo decisivo. Denunciare e dichiarare la violenza significa prendere posizione come soggetto di diritti che reclama giustizia, prendere la parola in termini legali ha un valore effettivo e simbolico nella definizione della vittima. Tuttavia la pressione sulla vittima a parlare degli eventi, di se stessa e della sua relazione con l'autore delle violenze e a segnalarlo alla legge, ha vaste implicazioni. Basandomi su di una ricerca svolta in Italia rifletto su come la violenza nelle relazioni di intimità può essere riportata alle istituzioni e come il soggetto maltrattato viene costruito in questo processo. Il governo di sé e la riflessività rendono la testimonianza comprensibile e legalmente rilevante e fanno delle donne delle vittime affidabili. Paradossalmente però la violenza esercitata in una relazione di intimità non prevede una vittima perfetta: questo soggetto affidabile richiesto dalle istituzioni non corrisponde alle esperienze, alle posizioni, alle decisioni, alle esitazioni delle donne.

In this article I wish to analyze the conundrums arising when battered women's words meet institutions and law. In the context of intimate partner violence documentary evidence is often not available and women's testimonies play an essential role. Pressing charges and speaking out means taking the floor as a legal subject in order to demand justice and to speak in legal terms has a decisive, effective and symbolic value in the definition of victimhood. Nonetheless the pressure on the victim to speak about the factual events, about herself and her relationship with the perpetrator and to denounce him before the law has vast implications. Drawing on a research carried out in Italy, I reflect on how violence in intimate relationships can be made present to the institutions and how the abused subject is constituted in front of them. Self-governance and reflexivity make testimony intelligible and legally significant and make women reliable victims. Yet paradoxically violence exerted in an intimate relationship does not easily provide for such a perfect victim: this reliable subject that institutions require does not match women's experiences, stances, decisions, hesitations.

\section{INDEX}

Parole chiave : violenza domestica, legge, istituzioni, genere, intimità

Mots-clés : violence conjugale, loi, institutions, genre, intimité

Keywords : domestic violence, law, institutions, gender, intimacy

\section{AUTEUR}

\section{ALESSANDRA GRIBALDO}

Dipartimento di Studi Umanistici, Università degli Studi Roma Tre alessandra.gribaldo@uniroma3.it 